\title{
TOPOLOGICAL SEQUENCE ENTROPY AND TOPOLOGICALLY WEAK MIXING
}

\section{Simin Li}

A characterisation of topologically weak mixing is given by using the topological sequence entropy.

\section{INTRODUCTION}

The notion of sequence entropy was first introduced by Kushnirenko [5]. Let $T$ : $(X, \mathcal{B}, \mu) \rightarrow(X, \mathcal{B}, \mu)$ be an invertible measure preserving transformation of a probability space and $A=\left\{t_{k}\right\}_{k=1}^{\infty}$ be a sequence of integer. Let $\mathcal{P}$ be the set of measurable partitions of $X$ with finite entropy. For $\xi \in \mathcal{P}$, define

$$
\begin{aligned}
h_{A}(T, \xi) & =\limsup \frac{1}{n} H\left(\bigvee_{i=1}^{n} T^{t_{i}} \xi\right) \\
h_{A}(T) & =\sup _{\xi \in \mathcal{P}} h_{A}(T, \xi) ;
\end{aligned}
$$

$h_{A}(T)$ is called the sequence entropy of $T$ with respect to the sequence $A$. When $A=\{k-1\}_{k=1}^{\infty}, h_{A}(T)$ is the usual entropy of $T$.

It is known that sequence entropy is an useful invariant of measurable dynamical systems and has close relationships with the spectrum of the systems. In $[4,6]$ the authors proved the following theorem.

TheOREM 1. Let $T:(X, \mathcal{B}, \mu) \rightarrow(X, \mathcal{B}, \mu)$ be an automorphism of a Lebesgue space. Then $T$ is weak mixing if and only if there exists an increasing sequence of natural numbers $A$ such that $h_{A}(T, \xi)=H(\xi)$ for all $\xi \in \mathcal{P}$.

On the other hand, topological entropy was also extended to topological sequence entropy by Goodman [3]. Let $X$ be a compact metric space and $f: X \rightarrow X$ be a continuous map. For an open cover $\alpha$ of $X$, denote by $N(\alpha)$ the minimal cardinality of any subcover of $\alpha$. The entropy $H(\alpha)$ of $\alpha$ is defined to be $\log N(\alpha)$. Let $A=\left\{t_{k}\right\}_{k=1}^{\infty}$ be a sequence of non-negative integers. We write

$$
h_{A}(f, \alpha)=\limsup _{n \rightarrow \infty} \frac{1}{n} H\left(\bigvee_{i=1}^{n} f^{-t_{i}} \alpha\right)
$$

\footnotetext{
Received 25th October, 2001
}

The author is supported in part by JSPS Postdoctoral Fellowship.

Copyright Clearance Centre, Inc. Serial-fee code: 0004-9727/02 \$A2.00+0.00. 
The topological sequence entropy of $f$ with respect to $A$ is defined by

$$
h_{A}(f)=\sup _{\alpha} h_{A}(f, \alpha)
$$

where $\alpha$ ranges over all open covers of $X$. When $A=\{k-1\}_{k=1}^{\infty}, h_{A}(f)$ is the topological entropy of $f$.

Let $f: X \rightarrow X$ be a continuous map of a compact metric space. Then $f$ is called topologically transitive if for any nonempty open sets $U, V$ of $X$, there exists an integer $n>0$ such that $U \cap f^{-n} V \neq \emptyset$. Also $f$ is called topologically weak mixing if $f \times f$ is topologically transitive. For a finite open cover $\alpha=\left\{U_{1}, U_{2}, \ldots U_{n}\right\}$ of $X$, define $I\left(U_{i}\right)=\left(\bigcup_{j \neq i} U_{j}\right)^{c}$. The cover $\alpha$ is called regular if $I\left(U_{i}\right)$ has nonempty interior for each $i$. It is easy to see that if an $n$-element open cover is regular then $N(\alpha)=n$, but the converse is not true. We shall prove the following theorem which is a topological version of Theorem 1.

THEOREM 2. Let $f: X \rightarrow X$ be a continuous map of a compact metric space. Then $f$ is topologically weak mixing if and only if for any regular open cover $\alpha$ of $X$, there exists an increasing sequence of non-negative integers $A=\left\{t_{k}\right\}_{k=1}^{\infty}$ such that $h_{A}(f, \alpha)$ $=H(\alpha)$.

\section{SOME LEMMAS}

LEMma 1. Suppose $\alpha=\left\{U_{1}, U_{2}, \ldots, U_{n}\right\}$ and $\beta=\left\{V_{1}, V_{2}, \ldots, V_{m}\right\}$ are regular open covers of the compact metric space. If $I\left(U_{i}\right) \cap I\left(U_{j}\right) \neq \emptyset$ for any $i, j$, then

$$
N(\alpha \vee \beta)=N(\alpha) N(\beta) .
$$

Proof: Obviously $N(\alpha \vee \beta) \leqslant N(\alpha) N(\beta)$. If the equality is not true, then there exists $i_{0}$ and $j_{0}$ such that $U_{i_{0}} \cap V_{j_{0}} \subset \bigcup \bigcup U_{i} \cap V_{j}$. Since $I\left(U_{i_{0}}\right) \cap U_{i}=\emptyset(i$ $\left.\neq i_{0}\right), I\left(V_{j_{0}}\right) \cap V_{j}=\emptyset\left(j \neq j_{0}\right)$, we have $(i, j) \neq\left(i_{0}, j_{0}\right)$

$$
\left(I\left(U_{i_{0}}\right) \cap I\left(V_{j_{0}}\right)\right) \cap\left(U_{i} \cap V_{j}\right)=\emptyset \text { if } \quad(i, j) \neq\left(i_{0}, j_{0}\right) .
$$

But this contradicts

$$
\emptyset \neq I\left(U_{i_{0}}\right) \cap I\left(V_{j_{0}}\right) \subset U_{i_{0}} \cap V_{j_{0}} \subset \bigcup_{(i, j) \neq\left(i_{0}, j_{0}\right)} U_{i} \cap V_{j}
$$

For a subset $Y$ of $X$, denote by $\operatorname{int}(Y)$ the interior of $Y$.

Lemma 2. Let $\alpha=\left\{U_{1}, \ldots, U_{n}\right\}$ and $\beta=\left\{V_{1}, \ldots, V_{n}\right\}$ be regular open covers of compact metric $X$. If $\operatorname{int}\left(I\left(U_{i}\right) \cap I\left(V_{j}\right)\right) \neq \emptyset$, then $\alpha \vee \beta$ is also a regular open cover.

PROOF: It follows immediately from that $I\left(U_{i} \cap V_{j}\right)=I\left(U_{i}\right) \cap I\left(V_{j}\right)$. 
LEMMA 3. ([2]) Let $f: X \rightarrow X$ be a continuous map of a compact metric space. If $f$ is topologically weak mixing, then $f \times f \times \ldots \times f$ ( $n$ times) is topologically transitive for any $n>0$.

LEMMA 4. ([1]) Let $f: X \rightarrow X$ be a continuous map of a compact metric space. If for any nonempty open sets $U, V$ of $X$, there exists an integer $n>0$ such that $U \cap f^{-n}(U) \neq \emptyset$ and $U \cap f^{-n}(V) \neq \emptyset$, then $f$ is topologically weak mixing.

\section{Proof of Theorem 2}

Proof: First suppose that $f: X \rightarrow X$ is topologically weak mixing and $\alpha$ $=\left\{U_{1}, U_{2}, \ldots, U_{n}\right\}$ is a regular open cover of $X$. Let $V_{i}=\operatorname{int}\left(I\left(U_{i}\right)\right)$. Then $V_{i}$ is a nonempty open set of $X$ as $\alpha$ is regular. Set $t_{1}=0$. Suppose

$$
0=t_{1}<t_{2}<\cdots<t_{k-1}
$$

have been defined and that $\bigvee_{i=1}^{k-1} f^{-t_{i}} \alpha$ is a regular open cover and $N\left(\bigvee_{i=1}^{k-1} f^{-t_{i}} \alpha\right)$ $=N(\alpha)^{k-1}$. For every $W_{j}^{\prime} \in \bigvee_{i=1}^{k-1} f^{-t_{i}} \alpha$, let $W_{j}=\operatorname{int}\left(I\left(W_{j}^{\prime}\right)\right)$. Then $W_{j}$ is nonempty as $\bigvee_{i=1}^{k-1} f^{-t_{i}} \alpha$ is regular. Since $f$ is topologically weak mixing, by Lemma 3 , there exists an integer $t_{k}>t_{k-1}$ such that $W_{j} \cap f^{-t_{k}} V_{i} \neq \emptyset\left(j=1,2, \ldots, n^{k-1}, i=1,2, \ldots, n\right)$. By Lemma 1 and Lemma $2, \bigvee_{i=1}^{k} f^{-t_{i}} \alpha$ is regular and $N\left(\bigvee_{i=1}^{k} f^{-t_{i}} \alpha\right)=N(\alpha)^{k}$. By induction we can choose an increasing sequence $A=\left\{t_{i} \mid i=1,2, \ldots\right\}$ such that, for any, $k>0$ we have $N\left(\bigvee_{i=1}^{k} f^{-t_{i}} \alpha\right)=N(\alpha)^{k}$ and therefore $h_{A}(f, \alpha)=\log N(\alpha)=H(\alpha)$.

Conversely if $f$ is not topologically weak mixing, then, by Lemma 4 , there exist nonempty open sets $U, V$ of $X$ such that for any $k>0$,

$$
U \cap f^{-k} U=\emptyset \text { or } U \cap f^{-k} V=\emptyset .
$$

Without loss of generality we may assume that $U \cap V=\emptyset$ (if $U \cap V \neq \emptyset$, we may replace $U, V$ by $U \cap V$ and $f^{-1}(U \cap V)$ respectively). Choose nonempty open sets $U_{1}, V_{1}$ of $X$ such that $\overline{U_{1}} \subset U, \overline{V_{1}} \subset V$. Let $U^{\prime}={\overline{U_{1}}}^{c}, V^{\prime}=\overline{V_{1}}$. Then $\alpha=\left\{U^{\prime}, V^{\prime}\right\}$ is a regular open cover and $U^{\prime} \supset U^{c}, V^{\prime} \supset V^{c}$. Now let $A=\left\{t_{i}\right\}_{i=1}^{\infty}$ be an arbitrary non-negative increasing sequence of integers. By $(*)$ we have that for any $m>0$,

$$
U \subset W_{0} \cap f^{-1} W_{1} \cap f^{-2} W_{2} \cap \ldots \cap f^{-m} W_{m},
$$

where

$$
W_{i}= \begin{cases}U^{\prime} & \text { if } \quad U \cap f^{-i} U=\emptyset \\ V^{\prime} & \text { if } \quad U \cap f^{-i} V=\emptyset\end{cases}
$$


Now given any $k>0$,

$$
U \subset f^{-t_{1}} W_{t_{1}} \cap f^{-t_{2}} W_{t_{2}} \cap \ldots \cap f^{-t_{k}} W_{t_{k}} .
$$

For $x \in U^{c}$, if there exists an $1 \leqslant j \leqslant k$ such that $f^{t_{j}}(x) \in U$, we let

$$
i=i(x)=\min \left\{j \mid 1 \leqslant j \leqslant k, f^{t_{j}}(x) \in U\right\} .
$$

Then

$$
\begin{aligned}
x \in & f^{-t_{1}} U^{\prime} \cap f^{-t_{2}} U^{\prime} \cap \ldots \cap f^{-t_{i-1}} U^{\prime} \cap f^{-t_{i}} U \\
& \subset f^{-t_{1}} U^{\prime} \cap f^{-t_{2}} U^{\prime} \cap \ldots \cap f^{-t_{i-1}} U^{\prime} \cap f^{-t_{i}}\left(W_{0} \cap f^{-\left(t_{i+1}-t_{i}\right)} W_{t_{i+1}-t_{i}}\right. \\
& \left.\cap f^{-\left(t_{i+2}-t_{i}\right)} W_{t_{i+2}-t_{i}} \cap \ldots \cap f^{-\left(t_{k}-t_{i}\right)} W_{t_{k}-t_{i}}\right) \\
= & f^{-t_{1}} U^{\prime} \cap f^{-t_{2}} U^{\prime} \cap \ldots \cap \cap f^{-t_{i-1}} U^{\prime} \cap f^{-t_{i}} W_{0} \cap f^{-t_{i+1}} W_{t_{i+1}-t_{i}} \cap \ldots \cap f^{-t_{k}} W_{t_{k}-t_{i}} \\
\in & \bigvee_{j=1}^{k} f^{-t_{j}} \alpha .
\end{aligned}
$$

If for any $1 \leqslant j \leqslant k, f^{t_{j}}(x) \notin U$, then

$$
x \in f^{-t_{1}} U^{\prime} \cap f^{-t_{2}} U^{\prime} \cap \ldots \cap f^{-t_{k}} U^{\prime} \in \bigvee_{j=1}^{k} f^{-t_{j}} \alpha
$$

Therefore

$$
N\left(f^{-t_{1}} \alpha \vee \ldots \vee f^{-t_{k}} \alpha\right) \leqslant k+2 \text { for any } k>0 .
$$

So $h_{A}(f, \alpha)=0$. It contradicts the assumption of the theorem.

Corollary 1. Suppose $X$ is a compact metric space which is not one point and $f: X \rightarrow X$ is topologically weak mixing. Then $\sup _{A} h_{A}(f)=\infty$ where the supremum is taken over all sequences of non-negative integers.

Proof: Since $f$ is topologically weak mixing and $X$ is not one point, $X$ is infinite. We can choose regular open covers of $X$ with $n$-elements for any $n>0$. By theorem 2, $\sup _{A} h_{A}(f)=\infty$.

EXAMPLE 1. The condition of the regularity of the cover of theorem 2 cannot be omitted. For example, let $X$ be a compact metric space which is not one point and $f: X \rightarrow X$ be a topologically weak mixing homeomorphism. Let $x_{0} \in X$ and $U_{1}=X-\left\{x_{0}\right\}$. Let $U_{2}$ be another open set of $X$ such that $\alpha=\left(U_{1}, U_{2}\right)$ is a regular open cover of $X$. It is easy to see that for any $k>0$ and any sequence $A=\left\{t_{i}\right\}_{i=1}^{\infty}$,

$$
N\left(f^{-t_{1}} \alpha \vee \ldots \vee f^{-t_{k}} \alpha\right) \leqslant k+1 .
$$

Therefore $h_{A}(f, \alpha) \neq H(\alpha)$. 


\section{REFERENCES}

[1] F. Blanchard, B. Host, and A. Maass, 'Topological complexity', Ergodic Theory and Dynamical Systems 20 (2000), 641-662.

[2] M. Furstenberg, 'Disjointness in ergodic theory, minimal sets, and a problem in Diophantine approximation', Math. Systems Theory 1 (1967), 1-49.

[3] T.N.T. Goodman, 'Topological sequence entropy', Proc. London Math.Soc. (3) 29 (1974), 331-350.

[4] P. Hulse, 'Sequence entropy and subsequence generators', J. London Math.Soc. (2) 26 (1982), 441-450.

[5] A.G. Kushnirenko, 'On metric invariants of entropy type', Russian Math. Surveys 22 (1967), 53-61.

[6] A. Saleski, 'Sequence entropy and mixing', J. Math. Anal. Appl. 60 (1977), 58-66.

Department of Mathematical Sciences

University of Tokyo

Komaba 3-8-1, Meguro Ku

Tokyo

Japan

e-mail: lisimin@ms.u-tokyo.ac.jp
Department of Mathematics

University of Science and Technology of China

Hefei

Anhui 230026

Peoples Republic of China 